\title{
Repenser l'éthique à travers l'imagination narrative et littéraire dans la pensée de Paul Ricœur et de Martha Nussbaum
}

\author{
Par ADÉLAÏDE GREGORIO FINS \\ Université de Paris 4 Sorbonne, Université de Coimbra
}

Résumé Cet article tente de démontrer l'apport de deux pensées philosophiques contemporaines à la question : que peut l'acte imaginaire? Paul Ricœur et Martha Nussbaum élaborent une théorie de l'imagination narrative et littéraire; le premier au sein de la phénoménologie herméneutique, la deuxième dans le cadre d'une philosophie d'inspiration libérale. Analysant le pouvoir sémantique du langage, plus précisément du récit et de la métaphore qui permettent d'imaginer le réel, cette recherche tente de comprendre la contribution de la littérature à la vie éthique. En effet, par l'articulation de la raison aux affects, on peut saisir la nature de la relation qu'entretient le sujetlecteur aux récits littéraires, rendant possible une compréhension plus fine de la complexité du réel. L'imagination constitue en effet chez ces deux philosophes le fil conducteur qui ouvre à l'altérité. C'est, semble-t-il, à cette condition qu'une éthique de la sollicitude peut se constituer.

\section{Introduction}

L'imaginaire joue un rôle important dans la vie des êtres humains, puisque c'est à travers lui que le sujet s'ouvre et oriente sa conscience vers d'autres modes possibles d'existence. Effectivement, nous connaissons tous des exemples où la force imaginative a conduit l'humanité vers le progrès, même si pendant des siècles, elle a semblé subordonnée aux prérogatives qui régissent le domaine de la connaissance. Galilée ne devient-il pas poète en comparant l'univers à un livre écrit en langage mathématique ? Cette métaphore, en proposant une nouvelle explication du monde, bien qu'extrava- 
gante et illusoire aux yeux de ses contemporains, révèle que l'imagination remplit une fonction heuristique qui ne se borne pas à illustrer des théories abstraites, mais donne à l'esprit humain la capacité de s'arracher aux visions naïves et périmées du réel. Partant de cet exemple, nous tenterons d'analyser la fonction heuristique du processus imaginatif mis en œuvre dans la littérature, en défendant l'idée que l'imagination narrative et littéraire constitue un langage, qui participe incontestablement au progrès moral et éthique de l'homme. Cependant, si l'acte d'imagination littéraire est le miroir de nos émotions et de nos sentiments, quelle valeur lui accorder ? Que font l'écrivain et le lecteur de toutes les images qui les habitent au contact de la littérature ? Cette visée imaginaire, est-elle toujours enrichissante, libératrice et créatrice, ou bien peut-elle nous enfermer dans une sorte d'aveuglement et d'aliénation nous privant de liberté, de jugement et d'autonomie ? Pouvonsnous soutenir que l'imagination littéraire conduit le sujet à mieux se connaître et à devenir éthiquement meilleur en extériorisant sa subjectivité qui favorise le dialogue et les relations intersubjectives? Par ailleurs, quelle influence les œuvres littéraires et les récits peuvent-ils avoir sur l'éthique, puisque l'imagination vagabonde selon une logique propre à chacun et est irréductiblement singulière?

Notre réflexion a pour objectif de montrer que l'imagination a toute sa place dans la vie rationnelle et dans la pensée philosophique du langage et des œuvres littéraires, telle que nous la trouvons dans les théories contemporaines de l'imaginaire élaborées par Paul Ricœur et Martha Nussbaum. En effet, l'objet de cette réflexion est d'examiner la contribution de la littérature à la philosophie morale et à l'éthique à travers le dialogue entre ces deux pensées qui postulent le fait que l'imaginaire dans la littérature produit un « libre jeu des possibilités » et de nouvelles manières d'être au monde, tout en formant un lieu où s'engendrent des représentations propices à inspirer des créations fondamentales pour l'existence de l'être humain. Si la littérature opère et enrichit le réel au travers des « variations imaginatives » (Husserl), elle révèle par la compréhension et l'interprétation à quel point l'imagination se situe à la charnière du théorique et du pratique, du sensible et de l'intelligible, de l'émotion et de l'abstraction, révélant ainsi la tension entre l'universel et le singulier.

Dans un premier temps, nous questionnerons le tournant herméneutique de la pensée de Paul Ricœur à travers la compréhension et l'interprétation des œuvres littéraires au sein du savoir scientifique ${ }^{1}$. L'œuvre de

${ }^{1}$ P. Ricœur, Le conflit des interprétations. Essais d'herméneutique, Paris, Seuil, 1969. 
Ricœur semble à cet égard fertile pour penser la création littéraire: le philosophe montre que la mise en intrigue du récit et l'imagination narrative jouent un rôle essentiel au plan à la fois individuel, intersubjectif et social. Il se produit dans ces conditions une reconfiguration de l'agir où la poïetique langagière (La métaphore vive) et la mimèsis produisent des connections et donnent du sens temporel aux actions humaines ${ }^{1}$. Dans son analyse herméneutique, la dialectique entre mêmeté et ipséité cherche à tracer le parcours de l'identité narrative et de l'éthique. En effet, l'imagination de l'identité narrative schématise (au sens kantien) et donc unifie : celle-ci apporte des motivations et structure l'action humaine en référence à des exigences éthiques, donnant naissance à un sujet actif qui affirme pouvoir se mettre à la place d'autrui et se révèle capable d'agir intentionnellement. Ce qui montre à quel point la fécondité de l'imagination narrative et éthique va de pair avec celle du langage. Il s'agit au fond de dépasser la conception d'un sujet trop sûr de lui-même pour donner naissance à un sujet-lecteur qui, à travers la capacité d'imagination, reconnaît sa fragilité, s'ouvre au monde et en fait la découverte par son rapport originaire à l'altérité. Nous ferons également appel à la réflexion éthique de Ricœur et à son herméneutique de l'Autre, pour mieux comprendre le sens des actions humaines qui se situent au cœur du récit, puisque c'est par la rencontre avec l'Autre que nous pouvons donner du sens à ce que nous vivons.

L'imaginaire, l'altérité et la question éthique de la sollicitude seront ainsi mises en dialogue dans un second temps de cette recherche avec la pensée de Martha Nussbaum. Tout comme Paul Ricœur, Martha Nussbaum pense que l'imagination littéraire enrichit le regard porté sur la vie humaine. La philosophe américaine réfléchit également sur le lien entre la capacité d'imagination et le rôle des émotions individuelles et publiques mobilisées par la littérature. Elle nous invite à penser la complexité du réel, non pas en référence à des abstractions, mais par une attention portée à la vie ordinaire et vulnérable, défendant une conception de la raison qui fait place à l'empathie et à la compassion, et plus largement à l'imagination. Articulant affects et raison, singularité et universel, elle défend une rationalité pratique, fondée sur une appréciation sensible des situations humaines particulières qui passe par un usage de l'imagination narrative telle qu'elle est mise en œuvre par la littérature. La narration et l'imagination dans ces textes cultivent les qualités de perception et de jugement moral essentielles pour assurer la circulation entre le moi privé et le nous public. Le récit littéraire et le travail de l'imagination constituent autant de moyens rendant possible notre parti-

${ }^{1}$ P. Ricœur, La Métaphore vive, Paris, Seuil, 1975. 
cipation à d'autres vies que les nôtres et nous font ressentir sur un mode singulier l'exigence universelle de justice. Martha Nussbaum élabore ainsi une pensée philosophique de la connaissance littéraire où l'imagination littéraire n'est pas opposée à l'argumentation rationnelle, mais peut au contraire lui apporter des éléments indispensables. Tout l'intérêt de la réflexion menée par Ricœur et Nussbaum est de fournir une issue à la double aporie d'un universel abstrait et d'un singulier livré à lui-même, en travaillant la tension qui unit les deux termes.

Mais, ce travail, il appartient au sujet-lecteur de le mener : en acceptant d'être porté par les mots, le lecteur doit apprendre à savoir se perdre pour mieux se retrouver au cours du récit : l'histoire des autres, il doit l'aborder comme si c'était la sienne pour, dans le même mouvement, laisser son imagination être travaillée par cette altérité. En ce sens, la double relation de l'œuvre au lecteur et du lecteur à l'œuvre constitue le point de départ de la contribution de la littérature à la formation et à la réflexion éthique. Il nous faut donc questionner le récit littéraire comme trace d'une présence et comme volonté de parler à distance, il s'agit de savoir comment un texte vit dans un auteur, dans un lecteur et comment celui-ci passe de l'un à l'autre. Ainsi la littérature ne désigne pas simplement un lieu d'accès à ce que la culture humaine peut produire de plus grand, mais constitue plus largement l'espace d'une circulation et d'une migration entre le même et l'autre, entre l'homogène et l'hétérogène, entre l'identité et la différence.

\section{Paul Ricœur}

Nous pensons que la théorisation de l'imaginaire constitue un fil conducteur de l'ensemble de l'œuvre ricœurienne, que le philosophe emprunte à partir des voies différentes de l'imagination créatrice, ou si l'on préfère, de la schématisation. Selon Ricœur, le contenu des images poétiques, littéraires et récits historiques comporte une pluralité de significations ; cependant, audelà de leurs sens immédiat, il faut interpréter les différents niveaux de signification pour rendre intelligibles ses images en dévoilant leur sens caché. Or la connaissance herméneutique favorise une interprétation qui échappe à la transparence. Ricœur parle explicitement d'imagination d'abord dans deux articles datant de 1976: "L'imagination dans le discours et dans l'action: pour une théorie générale de l'imagination » et "Idéologie et utopie : deux expression de l'imaginaire social », articles qui seront publiés 
plus tard dans le livre Du texte à l'action. Essais d'herméneutique II (1986) ${ }^{1}$. Par ailleurs, dans Réflexion faite ${ }^{2}$, Ricœur reconnait qu'une partie de son œuvre est consacrée à l'élaboration d'une philosophie de l'imagination. Cependant, à lire attentivement les œuvres du philosophe, nous remarquons que la question de l'imagination est étroitement liée à d'autres dimensions que le philosophe analyse comme celle de l'identité narrative et personnelle, de l'interprétation du temps, du sujet, de l'agir, de la sollicitude, du juste, etc. Le propre de Ricœur est d'envisager l'imagination non comme une faculté psychologique, mais comme un pouvoir sémantique : la métaphore et le récit permettent de percevoir le réel autrement qu'il n'est, donc de l'imaginer. L'image n'est pas moins que la perception et moins que le concept, elle est l'instrument qui permet leur articulation.

Cette promotion de l'imaginaire au rang de dimension pratique se vérifie dans la théorie ricœurienne de l'idéologie et de l'utopie. Ricœur cherche à construire une herméneutique du soi à travers la médiation productrice d'un composé hybride d'imagination, qui passe successivement par divers lieux de l'univers des sentiments et donne naissance à la subjectivité concrète. Les enjeux de la théorie ricœurienne de l'imagination à travers le concept d'identité narrative tel que nous le trouvons esquissé dans les trois tomes de Temps et récit ${ }^{3}$, et longuement développé dans la Sixième étude de Soi-même comme un autre ${ }^{4}$, ainsi que dans Du texte à l'action, Essai d'herméneutique II, mettent au jour la subjectivité humaine et la spécificité poético-pratique du soi de tout sujet agissant. Ainsi, récit historique et récit de fiction vont s'entrecroiser, faisant appel tous les deux à «l'intentionnalité de l'autre ». La littérature étant à ses yeux distanciation et appropriation médiatisée, nous sommes invités dans Temps et récit à penser la notion d'identité narrative au croisement entre histoire et fiction à travers le déploiement du temps.

Le philosophe va redéfinir et développer progressivement sa conception de l'identité narrative suivant la théorie des genres littéraires et la théorie de la lecture. Le cours qu'il dispense à l'Université de Chicago en 1975 montre la façon dont il interprète aussi bien la théorie kantienne de l'imagination productrice que la conception de l'imagination phénoméno-

\footnotetext{
${ }^{1}$ Ces deux articles sont la traduction française de Lectures on Ideology and Utopia, publié aux Etats-Unis en 1986, ce sont des notes de cours professées à l'université de Chicago en 1975 que le philosophe insère plus tard dans ce livre : P. Ricœur, $D u$ texte à l'action, Essais d'herméneutique II, Paris, Seuil, 1986.

${ }^{2}$ P. Ricœur, Réflexion faite, Paris, Éditions Esprit, 1995.

${ }^{3}$ P. Ricœur, Temps et récit, trois tomes, Paris, Seuil, 1983-1985.

${ }^{4}$ P. Ricœur, Soi-même comme un autre, Paris, Seuil, 1990.
} 
logique et analytique, révélant par ailleurs la faiblesse de leurs conceptions de l'imagination: elles n'ont pas réussi à mettre au jour la question de la fonction pratique de l'imagination si centrale dans la pensée ricœurienne. En effet, si nous trouvons chez Ricœur une fonction poétique de l'imagination, son article de 1976 intitulé "L'imagination dans le discours et dans l'action ${ }^{1}{ }^{1}$ montre qu'il y a également une dimension pratique de l'imagination, que Ricœur nomme "fonction du possible pratique », et qu'il caractérise en même temps comme une "fonction projective qui appartient au dynamisme même de l'agir ", capable d'éclairer, d'orienter et de dynamiser nos actions. Ces deux fonctions de l'imagination se trouvent alors articulées dialectiquement dans l'identité narrative. Ainsi, l'identité narrative va être le résultat de la production de l'imagination, d'un mixte d'histoire et de fiction, donnant à penser une identité dynamique qui échappe aux antinomies spéculatives posées par le problème de l'identité personnelle. La mise en récit apparaît donc comme la forme qui permet au sujet de rassembler sa propre vie aux prises avec les variations imaginatives et "l'unité narrative de la vie», expression de MacIntyre citée par Ricœur. Ainsi la vie peut être pensée, et donc imaginée de différents points de vue, suivant différentes trames et mises en intrigues, la mise en sens n'est ni unique ni univoque et la vie d'un sujet apparaît comme "enchevêtrée " dans l'histoire de la vie de ceux qui le côtoient. À la croisée entre histoire et fiction apparaît le récit de soi, berceau de l'identité narrative et de l'identité personnelle: le sujet va ainsi pouvoir créer une unité temporelle, rassembler les bribes de son histoire de vie et les évènements vécus.

Ce retour sur soi par un récit dans lequel il se reconnaît permet de conférer une unité et un fil conducteur à l'existence individuelle. À défaut d'être l'auteur effectif de tous les évènements de vie, le sujet devient le « coauteur» de leur sens dans une «recherche de cohérence ». Ce récit de vie est inachevé et sans cesse en mouvance, et même narré au passé, il offre aux évènements et aux expériences un caractère prospectif en révélant leurs attentes, par la manière dont il est mis en intrigue ici et maintenant il révèle la manière dont le narrateur est tourné vers l'avenir. Ainsi l'imagination poétique constitutive de nos identités narratives prend sa signification véritable lorsqu'elle se trouve réinscrite dans l'agir humain en devenir: c'est-à-dire que la constitution de nos identités narratives correspond davantage à une activité imaginative de construction pratique du soi humain qu'à une simple figuration fictionnelle; cette imagination pratique intègre et potentialise

${ }^{1}$ P. Ricœur, «L'imagination dans le discours et dans l'action», Du texte à l'action, Essais d'herméneutique II, Paris, Seuil, 1986, p. 237. 
l'imagination poétique ou représentative. Ce processus nous fait comprendre que la vie, y compris vulnérable, chemine en quête de son histoire de vie, de sa narration pour se comprendre soi-même et comprendre les autres. En outre, nous entrons dans l'éthique de la sollicitude par la confrontation à des valeurs transmises par les récits de vie, et notre jugement cherchera par ce biais à ancrer nos décisions dans un système de valeurs ; ainsi le bien-être humain, à côté du bonheur et du juste, constitue une valeur axiologique qui ne s'impose pas de l'extérieur ni en référence à une dimension universelle, mais qui vient du dedans, du ressentir du vouloir et du désir, un cheminement fait de responsabilité qui nous conduit de la communicabilité aux actions de soin envers autrui. Cette sollicitude qui s'adresse aux personnes dans leur singularité irremplaçable passe par la connaissance relationnelle des mots et du langage, à travers la littérature, le roman aussi bien que la poésie, et à travers elle, l'imagination donne aux êtres humains la possibilité de se mettre à la place d'autrui et de pouvoir se soucier des autres ${ }^{1}$. Or, justement, cette capacité d'initiative de l'homme au regard de son identité, à suivre Ricœur, va se former à partir d'un travail de subjectivité à l'œuvre pour donner un sens à la vie. L'activité narrative mise en évidence dans Soi-même comme un autre $^{2}$ se fixe sur l'unification biographique tournée vers le passé, où chacun peut se raconter l'histoire de sa vie, en recollant les morceaux épars. Et nous comprenons que c'est d'abord comme sujet parlant que notre autonomie apparaît fragilisée quand nous nous trouvons incapables de construire une identité narrative et de nous identifier à une histoire.

Tout au long de son œuvre, le philosophe nous invite à penser les capacités humaines : pouvoir d'agir, pouvoir de dire, pouvoir de se croire capable, pouvoir de faire le récit de sa vie. Il va donc rattacher l'identité narrative au thème de l'homme capable - l'homme agissant et souffrant -, homme à la fois porté au mal et capable du bien, celui qui se désigne comme un sujet d'action qui peut et qui fera le lien entre la vulnérabilité et l'autonomie. L'identité narrative fait le lien entre les différents aspects de ce que vit et éprouve le sujet et forme le support de ces possibilités d'action. Ce lien au niveau éthique va être travaillé par Ricœur dans son analyse de l'homme faillible ${ }^{3}$ à l'homme capable dévoilant les différents degrés de

\footnotetext{
${ }^{1}$ Voir également, S. Chavel, Se mettre à la place d'autrui, L'imagination morale, Rennes, PUR, 2011.

${ }^{2}$ P. Ricœur, Soi-même comme un autre, Paris, Seuil, 1990.

${ }^{3}$ Voir P. Ricœur, Philosophie de la volonté, tome 1, Le volontaire et l'involontaire, Paris, Aubier, 1950 ; Philosophie de la volonté, tome 2, Finitude et culpabilité, Paris, Aubier, 1960.
} 
capacités du soi dans son rapport à lui-même, à l'autre du face-à-face, à l'engagement pour le tiers, au mode herméneutique, à une autre langue que la sienne; un soi qui comporte des fractures et des fragilités mais qui rappelle la tâche sans fin qui consiste à raconter, à parler, à agir — un soi appelé à vivre une vie éthique. Tout en mettant l'accent sur l'imagination, l'autonomie et les capacités, la deuxième étude de son Parcours de la reconnaissance traite de la reconnaissance de soi-même et de l'autre en rapport avec la responsabilité humaine et interroge comment :

Le sujet d'action pourrait-il donner à sa propre vie une qualification éthique, si cette vie ne pouvait être rassemblée en forme de récit ? ${ }^{2}$

Ouvrant de plus en plus la philosophie aux ressources de la poétique, notamment dans Temps et Récit ${ }^{3}$, Ricœur donne un sens humain au temps et montre que c'est le langage le moins référentiel qui exprime le mieux l'agir humain, notamment parce que la mise en intrigue narrative et la métaphore rendent possible le « voir comme». Dans une dialectique de l'explication et de la compréhension langagière, le "voir comme» ouvre ainsi la voie à l'analyse de la fragilité. Ainsi, la réalité n'est en effet accessible que par le biais d'une mise en intrigue qui instaure une relation entre la narrativité et la temporalité, autrement dit par la combinaison de dimensions temporelles élaborées grâce à la triple mimèsis ${ }^{4}$. Le processus herméneutique est fondamental pour la constitution de l'identité narrative, définie comme la capacité de la personne à mettre en récit de manière concordante les événements douloureux de son existence. Ricœur parvient au concept d'identité narrative dans les conclusions de Temps et récit et la confrontation de ce terme avec les approches de l'identité personnelle développée par ses contemporains, Charles Taylor ${ }^{5}$ et Alasdair MacIntyre ${ }^{6}$, constitue en partie l'objet de Soimême comme un autre ${ }^{7}$. Ricœur y distingue trois composantes de l'identité personnelle : l'identité-idem ou mêmeté (ensemble des dispositions psychosociales par lesquelles on reconnaît un individu comme étant le même dans le temps), l'identité-ipse ou ipséité (maintien de soi par la parole donnée à

\footnotetext{
${ }^{1}$ Voir P. Ricœur, Soi-même comme un autre, Paris, Seuil, 1990.

${ }^{2}$ P. Ricœur, Parcours de la reconnaissance. Trois études, Paris Stock, 2004, p. 168.

${ }^{3}$ P. Ricœur, Temps et récit, trois tomes, Paris, Seuil, 1983-1985.

${ }_{5}^{4}$ Ibid.

${ }^{5}$ Voir C. Taylor, Les sources du moi. La formation de l'identité moderne, Paris, Seuil, 1989.

${ }^{6}$ A. MacIntyre, Après la vertu, Paris, PUF, 1981.

${ }^{7}$ Voir P. Ricœur, Soi-même comme un autre, Paris, Seuil, 1990.
} 
autrui) et l'identité narrative (capacité de mettre en récit de manière concordante les événements hétérogènes de notre existence). La notion d'identité narrative que Ricœur thématise dans le cadre d'une herméneutique du soi se résume au fait que je suis ce que je me raconte. Elle rejoint la définition de l'éthique de Soi-même comme un autre, qui adopte le point de vue téléologique d'Aristote, en tant que poursuite du bien, le point de vue déontologique kantien comme sens du devoir, et qui définit la "visée éthique » la visée de la "vie bonne », avec et pour autrui, dans des institutions justes » ${ }^{1}$.

D'après Ricœur, le rapport du lecteur à l'auteur se transforme quand la parole devient texte et trace: " l'écrit conserve le discours et en fait une archive (...) pour la mémoire individuelle et collective $»^{2}$. Si pour certains l'écriture est distance, détachement et abstraction, pour Ricœur le texte est une proposition de mise en relation et n'a de sens que par les résonnances qu'il provoque chez le lecteur. Cette relation comporte une multiplicité de formes de paroles portées par des individus ayant une intention. Pour qu'un texte puisse vivre, il doit être interprété et analysé par un lecteur actif et critique. Or il peut y avoir deux types de lecture ou d'approches : une approche formelle, objective, descriptive et distanciée, décrivant les structures grammaticales, et une approche interprétative. Ricœur propose de les combiner, laissant ainsi le lecteur achever le texte en le faisant résonner dans son expérience, de manière à lui procurer une signification personnelle et actuelle qui le concerne en propre. Par le texte qui vient d'ailleurs, de loin dans le temps et dans l'espace, le lecteur se met à mieux se comprendre luimême et donne un sens personnel et subjectif à ce qu'il lit. Mais ceci nous met devant deux cas de figure : celui du lecteur savant capable d'analyser, et celui du lecteur profane capable d'une réception subjective qui néanmoins réhabilite sa dignité puisque sa lecture fait partie du destin de l'œuvre. Il est nécessaire de prendre de la distance avec un texte, qui possède par ailleurs un sens, mais ce sens doit être interprété, commenté et formulé au travers d'un travail herméneutique qui motive l'action. Plonger dans la lecture des romans, pour Ricœur, n'implique pas de fuir le réel. Au contraire, cela consiste à créer un véritable lien entre lecture et action pour rentrer dans le réel ; en ce sens, lire signifie agir. Pour cela, le philosophe nous propose deux pistes : le lecteur s'approprie un texte pour pouvoir réfléchir aux actions personnelles, et à travers cette appropriation d'événements collectifs, il

\footnotetext{
${ }^{1}$ P. Ricœur, Soi-même comme un autre, Paris, Seuil, 1990, rééd. « Points-Essais » 2015, p. 202.

${ }^{2}$ P. Ricœur, Du texte à l'action, Essais d'herméneutique II, Paris, Seuil, 1986.
} 
manifeste son appartenance au groupe, à la société, à la nation et à l'histoire. Le fictionnel parle de la vie, de nos vies et du monde.

Les récits nous parlent d'actions commises par des personnages, des actions dont il nous faut comprendre les raisons et les motivations, des actions que nous comparons à celles que nous pourrions accomplir nousmêmes ou bien que nous refuserons. En ce sens, chaque récit réveille en nous nos motivations, nos dispositions, nos objectifs, nos intentions et jugements nous conduisant à délibérer intimement sur des situations proposées plus au moins proches de celles que nous vivons. Le récit narratif est imaginaire, il nous aide à construire nos comportements personnels puisque c'est une manière de travailler sur soi-même, la compréhension de soi et sa relation aux autres et au monde. Dans les trois tomes de Temps et récit, Ricœur établit une analyse entre la lecture du récit de fiction et celle du récit historique car le lecteur y projette dans les deux cas des affects. De fait, le récit historique comporte des «effets de fiction» malgré son attachement aux traces objectives. En effet, lorsque l'historien veut expliquer, il met en avant les motivations subjectives des personnages historiques, mais, contrairement au romancier, l'historien parle d'un temps collectif. Ainsi l'histoire « réinscrit le récit dans le temps de l'univers ${ }^{1}$, elle ne se préoccupe d'une destinée individuelle que pour la place qu'elle occupe dans une histoire commune. Le récit historique nous inscrit dans un temps long qui nous enserre et nous dépasse, un temps qui nous fait sentir la mort et la relativité de nos vies, un temps qui n'est pas uniquement le nôtre. Le temps historique produit un point de convergence entre le temps vécu, psychique et le temps universel, un « singulier collectif $»^{2}$. L'histoire offre ainsi une fonction poétique, créatrice qui nous permet de penser. Si la fiction nous interroge au plus près de la vie immédiate le récit vient nous chercher dans notre vie collective, construite au croisement de l'histoire et de la fiction et c'est à ce croisement que chacun construit son « identité narrative », identité que nous affinons, rationalisons à l'aide de l'imagination et au contact des récits fictionnels ou narratifs.

Nous venons de parcourir deux significations de la singularité, c'està-dire la présence du singulier dans les textes narratifs et historiques, ainsi que celle du singulier dans l'altérité qui est en constante tension avec l'universel. Cependant, Ricœur avance une troisième forme de singularité que

${ }^{1}$ P. Ricœur, Temps et récit, tome 3, Paris, Seuil, 1985, réed. « Points-Essais », 2005, p. 331 .

${ }^{2}$ P. Ricœur, Temps et récit, tome 3, Paris, Seuil, 1985, réed. « Points-Essais », 2005. Voir le dernier chapitre : "Vers une herméneutique de la conscience historique » $\mathrm{p}$. 374. 
nous avons vue un peu plus en haut, celle de «l'ipséité de soi » et qui révèle la manière dont la pensée ricœurienne théorise l'activité productrice de l'imagination.

Nous dirons pour conclure que l'imagination joue un rôle important dans la pensée de Paul Ricœur selon trois moments : téléologique, déontologique et de sagesse pratique.

Si la mise en récit intervient de façon essentielle dans la formation du sujet singulier, et si celle-ci constitue la condition intrinsèque du retour réflexif sur soi, la littérature aide dans le même mouvement à sortir de soimême, la lecture des romans nous mettant dans de meilleures dispositions pour imaginer et vivre potentiellement d'autres vies.

\section{Martha Nussbaum}

On peut faire l'hypothèse que Martha Nussbaum hérite de la théorie de l'imagination ricœurienne. Nous chercherons, dans cette perspective, à voir dans un deuxième moment de quelle manière la question de l'imaginaire se développe dans sa pensée philosophique. En effet, la philosophe américaine affirme, dans L'Art d'être juste, que «la lecture des romans apprend à imaginer des vies aux antipodes des siennes ${ }^{1}$. Celle-ci réfléchit également sur le lien entre la capacité d'imagination et le rôle des émotions individuelles et publiques. De fait, le livre a pour objet l'imagination littéraire et sa contribution à la vie publique. Dans celui-ci, elle s'adresse essentiellement à des futurs juges, pour lesquels la lecture de romans apporterait un éclairage bien particulier sur les situations humaines qui forment la matière de leur profession. La littérature, notamment romanesque, est donc une école de la singularité, aussi bien des personnes que des situations. En ce sens, les romans apprennent aux lecteurs à faire preuve d'imagination et d'empathie par le partage des sentiments des personnages : tout en se situant à distance des situations et des personnages dont il a connaissance, le lecteur s'identifie aux personnages et prend en compte le contexte de leur action sans pour autant tomber dans un relativisme sans relief. La réflexion sur la littérature narrative peut même, à suivre Nussbaum, contribuer à l'élaboration du droit : les raisonnements qui sont ceux du lecteur lorsque celui-ci lit un roman n'engagent pas que ses émotions et son intimité, l'espace privé du lecteur s'ouvrant ainsi sur l'espace public. Ainsi l'empathie et les émotions jouent

\footnotetext{
${ }^{1}$ M. Nussbaum, L'Art d'être juste (1995), trad. S. Chavel, Paris, Flammarion, coll. «Climats », 2015.
} 
un rôle essentiel dans le jugement rationnel à travers l'imagination, c'est-àdire la capacité de se mettre à la place de l'autre et de comprendre la situation objective dans laquelle il se trouve, qualité requise dans le travail qui est celui du juge. Nussbaum fait le lien entre la littérature et les émotions en faisant ressortir le caractère rationnel des émotions. Par la narration littéraire, nous développons notre imagination, ce qui nous permet de cultiver nos capacités à percevoir ce que d'autres peuvent ressentir, et par là même à nous enrichir. Citant un texte de J-J. Rousseau, Emile ou de l'éducation, au livre $\mathrm{IV}^{1}$, Nussbaum souligne que la non-reconnaissance de sa propre vulnérabilité amène à l'aveuglement et à l'indifférence vis-à-vis l'Autre. Il faut donc pouvoir imaginer ce que c'est la faim, la soif, la misère pour être en mesure de comprendre ce qu'éprouvent ceux qui souffrent. Cette importance que le roman peut apporter à la "vie ordinaire" (terme wittgensteinien) donne chair au conflit entre l'imagination littéraire et la conception utilitariste dans l'analyse de Temps difficiles de Charles Dickens (1854), que Nussbaum traite au sein du chapitre « Des poètes pour juge».

Le récit romanesque pour Martha Nussbaum contient une dimension politique et morale, en ceci qu'il suscite chez le lecteur une propension à se montrer attentif à la complexité de la vie et l'invite par là à un plus grand respect des valeurs démocratiques. L'ouvrage postule que juges et lecteurs devraient avoir en commun d'être des spectateurs empathiques et impartiaux par le biais d'une raison ouverte aux émotions. L'acte de lire revêt donc une valeur morale parce qu'il exige à la foi immersion et conversion critique ; on confronte sa lecture à sa propre expérience et on la confronte aussi aux expériences des autres. En ce sens, la littérature romanesque forme la dimension à travers laquelle l'idée du " cogito brisé » de Ricœur prend toute sa valeur: ce que je suis n'a de portée effective et de signification éthique que si j'accepte de me laisser travailler par l'altérité, mon identité existentielle ne prenant sens que dans le rapport constitutif que j'entretiens à la singularité des autres, que je n'ai d'ailleurs pas besoin de connaître personnellement en tant que ce sont des vies singulières anonymes. Nussbaum nous conduit par ce biais à repérer la tension qui existe entre le singulier et l'universel : je ne peux saisir le caractère singulier des existences auxquelles m'introduit la lecture des romans que si je suis en mesure de les référer à l'universel qui les traverse: Temps difficiles, de Dickens, ou encore Les raisins de la colère, de Steinbeck, nous révèlent ce que les sociétés modernes peuvent générer en termes d'injustice parce que notre imagination fait le va et vient entre le contexte singulier des situations vécues et l'universalité des

${ }^{1}$ J.-J. Rousseau, Emile ou de l'éducation, Paris, GF-Flammarion, 1966. 
principes de justice. Si nous n'étions pas sensibles à la particularité de la situation vécue par un personnage de roman, notre rapport à l'universalité de la morale resterait abstrait ; à l'inverse, si nous ne faisions pas référence à un universel normatif dépassant la multiplicité des situations d'injustice, nous ne pourrions pas être choqués par leur caractère injuste précisément, car nous ne repérerions même pas la contradiction entre l'exigence universelle de justice et le fait qu'une situation n'est pas conforme à une telle exigence.

Nussbaum propose une réflexion sur la justice en référence à la conception aristotélicienne de la personne, afin de dépasser la conception de la personne pensable dans les termes rationnels que Rawls utilise dans sa Théorie de la justice (1971) ${ }^{1}$. Or, selon Nussbaum, la vie humaine est un mélange d'émotions, d'imagination et de rationalité qui peuvent être affectées par le temps et les aléas de la fortune, d'où l'importance d'intégrer dans le contrat de justice les dimensions de vulnérabilité et de dépendance. Dans Frontiers of Justice ${ }^{2}$, Nussbaum propose une conception renouvelée de la justice en défendant l'autonomie personnelle et l'accès équitable de chacun à un ensemble de " capacités » humaines en rapport avec la vulnérabilité qui habite les êtres humains. L'idée de «capacité » que Nussbaum présente comme conception du bien humain et finalité de la justice politique permet une plus grande compréhension de la vie humaine que celle du modèle libéral rawlsien. Elle présente une liste de dix « capacités » humaines de base, qui rend compte de la conception normative de la vie humaine, et où la bonne "autonomie personnelle» se fonde sur la bonne vulnérabilité. La théorie des " capacités » permet l'extension de la notion de droit et permet de concevoir une rationalité pratique et émotionnelle à la fois active et vulnérable, ouvrant un immense champ de réflexion sur la place des émotions et de l'imagination sur l'éducation, le droit et l'action publique. Si Nussbaum situe le débat de sa théorie des « capacités » par rapport aux théories de la justice, notamment la Théorie de la justice de Rawls (1971), c'est pour en faire une critique du Moi rationaliste et compact, coupé de toute relation, ne donnant pas d'importance au Moi relationnel, ni à l'imagination, ni à la vulnérabilité du corps ou encore au travail du soin.

\footnotetext{
${ }^{1}$ J. Rawls, Théorie de la justice, 1971, trad. C. Audart, Paris, Seuil, 1987, rééd. « Points-Essais », 1997.

${ }^{2}$ M. Nussbaum, Frontiers of Justice. Disability, Nationality, Species Membership, Cambridge, Massachusetts, Harvard University Press, 2006. Tout en lui donnant une autre consistance, l'auteur développe l'approche des « capacités » que Sen présente dans L'Idée de Justice, Paris, Flammarion, 2009.
} 
Par ailleurs, la sollicitude et l'attention portées à autrui sont ici des composantes essentielles pour l'élaboration éthique. La philosophe américaine utilise le terme de sollicitude à la place du care, ce qui fait penser qu'il s'agit donc d'une théorie éthique du care qui ne dit pas son nom, mais qui insiste toutefois sur les capacités des êtres humains plutôt que sur leurs fonctions, comme c'est le cas dans la justice procédurale de Rawls. En effet, sa démarche est très différente des autres théoriciennes du care; nous trouvons chez Nussbaum des êtres humains dotés des capacités rationnelles et relationnelles capables de distinction entre moralité et convention, alors que les petites filles des enquêtes quantitatives et qualitatives de Gilligan en semblent dépourvues ${ }^{1}$. Ainsi l'existence d'une orientation morale vers le juste - ou l'attention pour soi-même et pour autrui — n'est donc pas guidée par la question du genre féminin ou masculin, mais résulte d'une maturité qui advient au contact de la littérature, où l'imaginaire et la réflexion contribuent à l'équilibre entre la justice et le soin. Cette position diffère également de la philosophie de Tronto, puisque chez Nussbaum les émotions font appel à l'imagination, imagination qui fait référence à l'autre ${ }^{2}$. De plus, il y a chez elle une réflexion morale sur les relations dans le champ du privé susceptibles de nourrir l'approche de la justice et du bien public ${ }^{3}$. Nussbaum, comme Ricœur, discute avec le positivisme juridique et la théorie de Justice de Rawls, notamment dans son livre Poetic Justice ${ }^{4}$. Tout comme Paul Ricœur, Martha Nussbaum pense que l'identité narrative et littéraire enrichit le regard porté sur la vie humaine à travers l'idée d'une hospitalité possible que la littérature offre aux sentiments et aux émotions grâce au médium de l'imagination qui rend possible l'éthique et la morale.

En définitive, la littérature nous permet de comprendre l'acte d'imagination afin de définir l'altérité de l'autre, la sollicitude, le soi, l'amour et la justice. Cet acte imaginaire comporte deux fonctions essentielles : la fonction créatrice d'un monde meilleur et la fonction libératrice de l'ignorance, de l'injustice, de l'indifférence et du malheur. Or, Ricœur parle d'un sentiment d'injustice, pas du désamour ni de l'amour brisé et nous y trouvons une réflexion sur la justice, mais pas sur l'amour. Alors que chez Nussbaum

\footnotetext{
${ }^{1}$ C. Gilligan, Une voix différente. Pour une éthique du care, trad. S. Laugier, Paris, Flammarion/Champs, 2008.

${ }^{2} \mathrm{~J}$. Tronto, Un monde vulnérable. Pour une politique du care, trad. H. Maury, Paris, La Découverte, 2003.

3 Voir à ce sujet M. Nussbaum, La connaissance de l'amour. Essais sur la philosophie et la littérature (1991), trad. S. Chavel, Paris, Le Cerf, 2010.

${ }^{4}$ M. Nussbaum, L'Art d'être juste (1995), trad. S. Chavel, Paris, Flammarion, coll. « Climats », 2015.
} 
l'acte d'imagination peut demander amour et justice. Cette contribution révèle à quel point l'analyse de l'imaginaire dans les œuvres de ces deux philosophes contribue à la reconnaissance de soi et de l'autre : dans l'herméneutique de Paul Ricœur à travers l'identité narrative du " cogito brisé » de l'homme faillible et la sagesse pratique de l'homme capable ; dans la théorie du care de Martha Nussbaum, à travers l'éducation à la vertu par l'imagination littéraire. Deux philosophies de la sollicitude indispensables au bien-être et au vivre ensemble et qui contribuent à l'édifice de la pensée éthique et politique contemporaine.

\section{Bibliographie}

Aristote, L'Éthique à Nicomaque, trad. J. Tricot, Paris, Vrin, 1997.

Bouriau, C., Qu'est-ce que l'imagination ?, Paris, Vrin, 2003.

Chavel S., Se mettre à la place d'autrui, L'imagination morale, Rennes, PUR, 2011.

Gilligan, C., Une voix différente. Pour une éthique du care, trad. S. Laugier, Paris, Flammarion/Champs, 2008.

Jonas, H., Le principe responsabilité, trad. J. Greisch, Paris, Flammarion, 2008.

Kymlicka, W., Les théories de la justice. Une introduction, trad. M. Saint-Upéry, Paris, La Découverte, 1999.

Laugier, S., (dir.), Éthique, littérature, vie humaine, Paris, PUF, 2006

MacIntyre, A., Après la vertu, Paris, PUF, 1981.

Nussbaum M., Frontiers of Justice. Disability, Nationality, Species Membership, Cambridge, Massachusetts, Harvard University Press, 2006.

- La connaissance de l'amour. Essais sur la philosophie et la littérature (1991), trad. S. Chavel, Paris, Le Cerf, 2010.

- L'Art d'être juste (1995), trad. S. Chavel, Paris, Flammarion, coll. «Climats», 2015.

Rousseau, J.-J., Emile ou de l'éducation, Paris, GF-Flammarion, 1966.

Ricœur, P., Philosophie de la volonté, tome 1, Le volontaire et l'involontaire, Paris, Aubier, 1950.

- Philosophie de la volonté, tome 2, Finitude et culpabilité, Paris, Aubier, 1960.

— Le conflit des interprétations. Essais d'herméneutique, Paris, Seuil, 1969.

- La Métaphore vive, Paris, Seuil, 1975.

- Temps et Récit, t. 1, L'intrigue et le récit historique, Paris, Seuil, 1983.

- Temps et Récit, t. 2, La configuration du temps dans le récit de fiction, Paris, Seuil, 1985.

- Temps et Récit, t. 3, Le temps raconté, Paris, Seuil, 1985.

— Du texte à l'action, Essais d'herméneutique II, Paris, Seuil, 1986.

- Soi-même comme un autre, Paris, Seuil, 1990.

— Réflexion faite, Paris, Éditions Esprit, 1995. 
- Le juste 1, Paris, Éditions Esprit, 1995.

- Le Juste 2, Paris, Éditions Esprit, 2001.

- Parcours de la reconnaissance. Trois études, Paris, Stock, 2004.

Rawls, J., Théorie de la justice, 1971, trad. C. Audart, Paris, Seuil, 1987, rééd. « Points-Essais », 1997.

Sen A., L'Idée de Justice, Paris, Flammarion, 2009.

Taylor C., Les sources du moi. La formation de l'identité moderne, Paris, Seuil, 1989.

Tronto J., Un monde vulnérable. Pour une politique du care, trad. H. Maury, Paris, La Découverte, 2003. 